\title{
Weibull model of Multiplicity Distribution in hadron-hadron collisions
}

\author{
Sadhana Dash*1] and Basanta K. Nandi \\ *Indian Institute of Technology Bombay, Mumbai, India
}

\begin{abstract}
We introduce the Weibull distribution as a simple parametrization of charged particle multiplicities in hadron-hadron collisions at all available energies, ranging from ISR energies to the most recent LHC energies. In statistics, the Weibull distribution has wide applicability in natural processes involving fragmentation processes. This gives a natural connection to the available state-of-the-art models for multi-particle production in hadron hadron collisions involving QCD parton fragmentation and hadronization.
\end{abstract}

\section{Introduction}

Inclusive charged particle multiplicity distribution had been extensively studied for a long time in hadronic collisions. The multiplicity distribution characterizes the hadronic final state and provides important insights on the production mechanism of muti-particle final states. In particular, they are sensitive to the underlying QCD dynamics in hadron-hadron collisions. The previous phenomenological studies of multiplicity distributions at various energies have been done in terms of parameters of the Negative Binomial Distribution (NBD) function. Although the interpretation of the NBD in terms of particle production is not fully understood, it remarkably described the data at lower energies [1. However, deviations from the NBD were observed at higher energies [1, 2, 3] which puts constraints on its universal applicability for wider range of energies. It was followed by two-component model of particle production, interpreting one as the soft and the other as the semi-hard component [4]. This led to the description of the data by the weighted combination of two NBD's. The multiplicity distributions at LHC energies and for all pseudorapidity intervals were well described by this approach [2, 5]. However, it was pointed out that the excellent description of multiplicity distributions of hard QCD events in larger $\eta$ window of $p-p$ collisions at $7 \mathrm{TeV}$ contradicts the very concept of two component model [5. A very detailed discussion on multiplicity measurements and various approaches can be found at [6]. In this work, we introduce a statistical distribution, namely, the Weibull distribution [7] which we found to describe the $p-p(p-\bar{p})$ data at all available energies ranging from ISR to LHC. In addition, this distribution gives an appealing physical interpretation.

\footnotetext{
${ }^{1}$ email: sadhana@phy.iitb.ac.in
} 


\section{Weibull distribution}

Many evolving systems in nature exhibit skewed distributions and among them Weibull and lognormal distributions appear more ubiquitously in a variety of systems 7, 8. In particular, the Weibull distribution is widely used to describe size distribution obtained in diverse fields such as material fragmentation [9, cloud droplets 10, biological systems 11, 12, wind speeds 13 and extreme value statistics [14, 15] etc. Since final state particle multiplicity can be seen as evolved from initial hadron-hadron collisions, one can expect to use the Weibull distribution to describe the probability of producing charged particles.

The probability distribution of a continuous random variable ' $n$ ' in terms of two parameter Weibull distribution is given by

$$
P(n, \lambda, k)=\frac{k}{\lambda}\left(\frac{n}{\lambda}\right)^{k-1} e^{-(n / \lambda)^{k}}
$$

where $k$ is the shape parameter and $\lambda$ is the scale parameter of the distribution. The mean of the distribution is given in terms of $\lambda$ and $\mathrm{k}$ as,

$$
\langle n\rangle=\lambda \Gamma\left(1+\frac{1}{k}\right)
$$

The description of a physically based derivation of Weibull distribution with respect to fragmentation processes can be found at [7]. In this particular work [7, it was shown that the result of a single event fragmentation leading to a branching tree of cracks in the material that show fractal behaviour can be described by Weibull distribution. This can be related to the mass distribution or particle number distribution developed by fragmentation process. It was also shown that a particular mass distribution closely resembles the lognormal distribution which was quite popular for sometime describing fragmentation distributions [7]. The lognormal distribution also described well the particle multiplicities at ISR energies [16].

The underlying mechanism of particle production in hadronic collisions as given by current models of particle production is based on the fragmentation of partons into observed hadrons. Irrespective of particular details, most of the models of multi-particle production contain an evolution composed of various steps that are based on previous intermediate steps and are influenced by the same. The steps involving hard and semi-hard processes are well explained by perturbative quantum chromodynamics (QCD) [17, 18, 19] while models for soft interactions involve large class of string fragmentation models [20, 21]. Regardless of differences in details, the cascade nature of models involving fragmentation of partons is apparent and thus one can use Weibull distribution to model the basic multiplicity distribution in hadron-hadron collisions. This also corroborates to the clan model involving particle cascades as mechanism of particle production [2, 4]. 

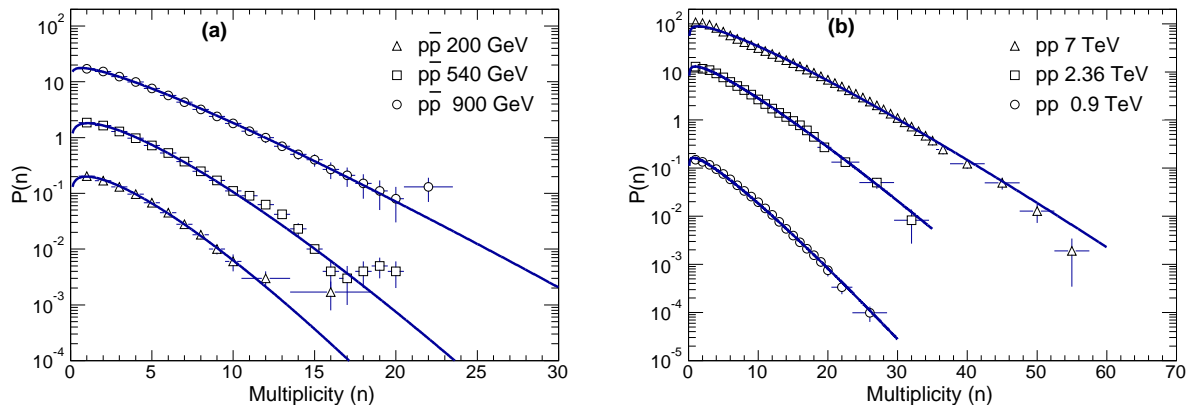

Figure 1: (a) UA5 measurement of charged particle multiplicity distribution in $p-\bar{p}$ collisions at mid-rapidity at $\sqrt{s_{N N}}=200 \mathrm{GeV}, 540 \mathrm{GeV}$ and $900 \mathrm{GeV}$ [1].(b) CMS measurement of multiplicity in $p-p$ collisions at midrapidity at $\sqrt{s_{N N}}=0.9 \mathrm{TeV}, 2.36 \mathrm{TeV}$ and $7 \mathrm{TeV}[22$. The solid line represents the Weibull fit to the data points. The data points for a given energy are appropriately scaled for better visibility.

\section{Multiplicity Distribution and Weibull Pa- rameters}

In the present scenario where we have experimental data on multiplicity distributions in widest range of energies and pseudorapidity intervals, it is worth trying to check whether we can parametrize the data in terms of Weibull parameters. We identify the variable $n$ with the charged particle multiplicity and perform the fits to the data points using the chi-square minimization method. We fitted the multiplicity distribution in $p-\bar{p}$ and $p-p$ collisions as measured by UA5 experiment at SPS energies [1] and by CMS experiments [17 at LHC energies, respectively. Figure 1(a) shows the Weibull fits to the multiplicity distribution at mid-rapidity for the $p$ $\bar{p}$ collisions as measured by UA5 experiments at $200 \mathrm{GeV}, 540 \mathrm{GeV}$ and $900 \mathrm{GeV}$. The fits to the recent multiplicity distributions in $p-p$ collisions as measured by the CMS experiment at $900 \mathrm{GeV}, 2.36 \mathrm{TeV}$ and $7 \mathrm{TeV}$ are shown in Figure 1(b). As can be seen from the figures, the Weibull fit gives an excellent description of the data at all energies. The values of the parameters and the chi-square of the fit is tabulated in Table 1. We can easily compare that the parameters obtained from the fits at $p-\bar{p}$ and $p$ - $p$ collisions at $900 \mathrm{GeV}$ (from two different experiments) are comparable within errors. Table 1 lists all the parameters obtained from the Weibull fit together with the chi-square and extracted mean multiplicity for different collision systems studied.

At LHC energies, the multiplicity distribution as measured by LHCb experiment covers the widest range of pseudorapidity intervals at $7 \mathrm{TeV}$ 23]. The single NBD distribution could not give a good description of the data in different pseudorapidity intervals [5]. Figure 2(a) shows the fit for the $\mathrm{LHCb}$ data for various pseudorapidity intervals in $p$ - $p$ collisions at 7 


\begin{tabular}{|c|c|c|c|c|}
\hline \hline Collision systems & $k$ & $\lambda$ & $\chi^{2} / n d f$ & $\langle n\rangle$ \\
\hline$p-\bar{p}(200 \mathrm{GeV})$ & $1.27 \pm 0.04$ & $3.17 \pm 0.07$ & 0.54 & $2.93 \pm 0.029$ \\
\hline$p-\bar{p}(540 \mathrm{GeV})$ & $1.26 \pm 0.01$ & $3.58 \pm 0.04$ & 1.66 & $3.32 \pm 0.015$ \\
\hline$p-\bar{p}(900 \mathrm{GeV})$ & $1.11 \pm 0.01$ & $4.07 \pm 0.08$ & 0.33 & $3.91 \pm 0.015$ \\
\hline$p-p(0.9 \mathrm{TeV})$ & $1.13 \pm 0.01$ & $4.17 \pm 0.06$ & 0.03 & $3.98 \pm 0.018$ \\
\hline$p-p(2.36 \mathrm{TeV})$ & $1.14 \pm 0.03$ & $5.41 \pm 0.15$ & 0.22 & $5.15 \pm 0.036$ \\
\hline$p-p(7 \mathrm{TeV})$ & $1.15 \pm 0.01$ & $7.35 \pm 0.16$ & 0.85 & $6.98 \pm 0.026$ \\
\hline \hline
\end{tabular}

Table 1: List of parameters and chi-square values of the Weibull fit to the data for various collision systems.
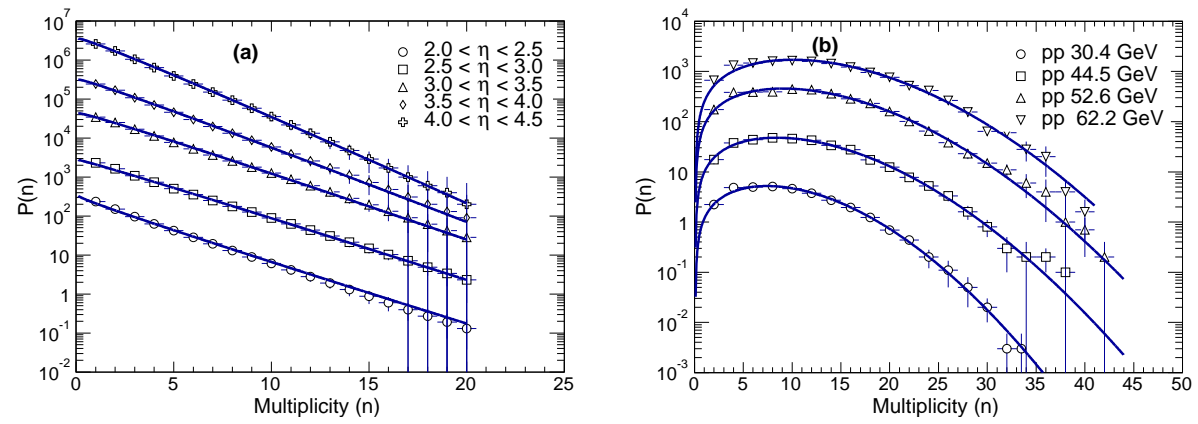

Figure 2: (a) LHCb [18] measurement of charged particle multiplicity distribution in $p-p$ collisions at different pseudorapidity intervals at $\sqrt{s_{N N}}=$ $7 \mathrm{TeV}$ (b) ISR measurement of topological cross-sections as a function of multiplicity in $p-p$ collisions at various energies [19. The solid line represents the Weibull fit to the data points in (a) and (b). The data points for a given energy are appropriately scaled for better visibility.

$\mathrm{TeV}$. The data are remarkably described by the Weibull distribution in all the pseudorapidity intervals. The applicability was also checked at lower energies measured by ISR experiment [24] as shown in Figure 2(b). This figure provides an excellent comparison where we observe that the Weibull distribution successfully explains the data at two diverse energy domains.

The energy dependence of the mean charged multiplicity reflects the underlying particle production mechanism. Feynman scaling predicts that the total mean number of particles produced obeys an energy dependence proportional to $\ln \left(\sqrt{s_{N N}}\right)$ 25. Figure 3(a) shows the mean multiplicity as a function of the center of mass energy. As can be seen from the figure, the variation of mean multiplicity with beam energy can be quantified by the expression

$$
\langle\mathrm{n}\rangle=\mathbf{A}+\mathbf{B}\left[\ln \left(\sqrt{s_{N N}}\right)\right]+\mathbf{C}\left[\ln ^{2}\left(\sqrt{s_{N N}}\right)\right]
$$

We also observe that the scale parameter, $\lambda$ shows an energy dependence which is similar to that of the mean multiplicity . This is shown in Figure 

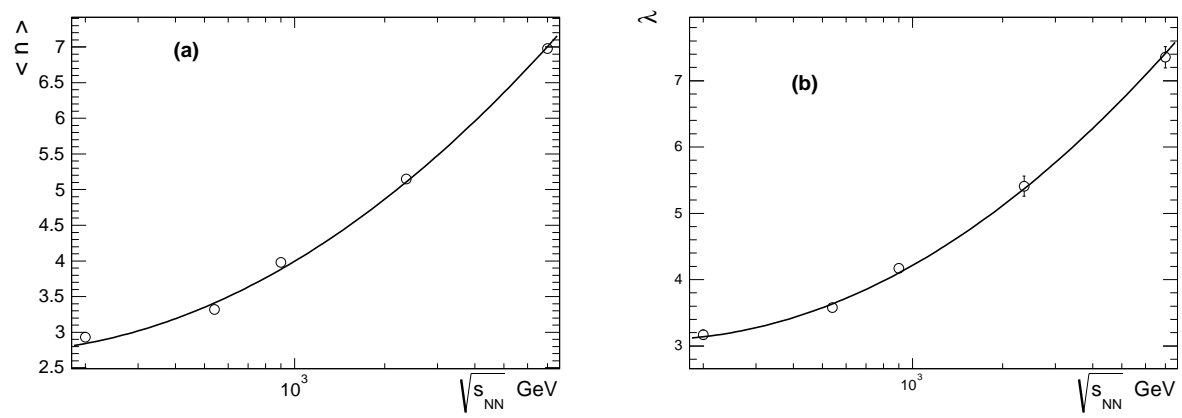

Figure 3: (a) The variation of mean multiplicity at mid-rapidity as a function of center of mass energy. (b) The variation of $\lambda$ parameter extracted from the Weibull fit as a function of beam energy The solid line represents the fit given by expression (3) to the data points.

3(b). We can see from Table 1 that the values of the shape parameter $k$ do not vary significantly with the center of mass energy. Taking this into account and the extrapolated values of the $\lambda$ parameter, we predict the multiplicity distribution at mid-rapidity in $p$ - $p$ collisions at the upcoming $\mathrm{LHC}$ run at $\sqrt{s_{N N}}=5.5 \mathrm{TeV}(\lambda=6.88, \mathrm{k}=1.15)$ and $14.0 \mathrm{TeV}(\lambda=$ $9.02, \mathrm{k}=1.15)$. Figure 4 depicts the predicted multiplicity distribution at $\sqrt{s_{N N}}=5.5 \mathrm{TeV}$ and $14.0 \mathrm{TeV}$ at mid-rapidity. The mean multiplicity, $\langle n\rangle$, at $5.5 \mathrm{TeV}$ and $14.0 \mathrm{TeV}$ turns out to be 6.54 and 8.56, respectively. The measurement of multiplicity distribution at larger $\sqrt{s_{N N}}$ at LHC will be a first test of the further applicability of the Weibull distribution and will add credibility to the extrapolation.

\section{Summary and Remarks}

We have demonstrated that the Weibull distribution provides an excellent description of the multiplicity distributions of inclusive charged particles in hadronic collisions at all available energies and at all pseudorapidity intervals. This is particularly significant since the Weibull distribution arises in cascade processes involving fragmentation of the source. This leads to a very interesting physics interpretation in terms of current dynamical models of multi-particle production. Because of the universal nature of the parton emission and hadronization process, we claim that the Weibull distribution gives the most proper description compared to the previous distributions used in multi-particle production processes.

\section{References}

[1] R. E. Ansorge et al., [UA5 Collaboration], Z.Phys. C. 43, 357 (1989).

[2] P. Ghosh, Phys.Rev. D 85, 054017 (2012). 


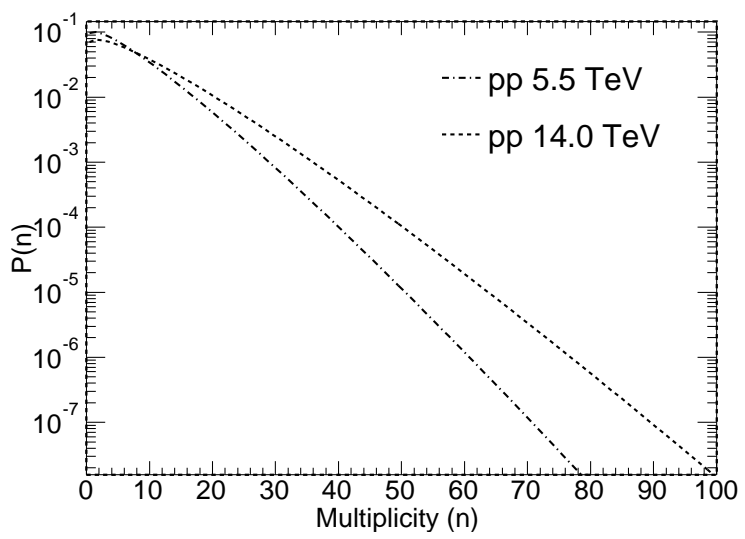

Figure 4: The mid-rapidity charged particle multiplicity distribution in $p$ $p$ collisions at $\sqrt{s_{N N}}=5.5 \mathrm{TeV}$ and $14.0 \mathrm{TeV}$ as predicted by Weibull parametrization.

[3] F. R. et al., [CDF Collaboration], Aspen Multipart. Dyn. 1993 , 400 (1993).

[4] A. Giovannini and R. Ugoccioni, Phys.Rev. D 60,074027 (1999).

[5] P. Ghosh and S. Muhuri, Phys.Rev. D 87, 094020(2013).

[6] J. F. Grosse-Oetringhaus and K. Reygers, J.Phys.G:nucl.Part.Phys. 37, 083001 (2010).

[7] W. Weibull, J. Appl. Mech. 18, 293 (1951).

[8] M. Y. Choi, H. Choi, J. Y. Fortin and J. Choi, EuroPhys. Lett.

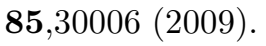

[9] W. K. Brown, and K. H. Wohletz, J. Appl. Phys. 78, 2758 (1995).

[10] Y. Liu and J. Hallett, J. Atomos. Sci. 55, 527(1998).

[11] J. Jo, M. Y. Choi and D. S. Koh, Biophys. J. 93,2655 (2007).

[12] J. Jo, J. Y. Fortin and M. Y. Choi, Phys. Rev. E 83, 031123 (2011).

[13] E. S. Takle and J. M. Brown, J. Appl. Meteor. 17, 556 (1978).

[14] E. Bertin and M. Clusel, J. Phys. A 39, 7607 (2006).

[15] N. R. Moloney and J. Davidsen, Phys. Rev. E 79, 041131 (2009).

[16] S. Carius and G. Ingelman, Phys. Lett. B., 252, 647-652 (1990).

[17] T. K. Gaisser, F. Halzen, A. D. Martin and C. J. Maxwell, Phys. Lett. B., 116, 219 (1986) 
[18] T. Sjostrand and M. van Zijl, Phys. Rev. D 36., 2019, (1987).

[19] X. N. Wang, Phys. Rev. D 43, 104 (1991).

[20] A. Capella, U. Sukhatme, C. I. Tan and J. Tran Thanh Van, Phys. Rept. 236225 (1994).

[21] A. B. Kaidalov Phys. Atom. Nucl, 661994 (2003).

[22] V. Khachatrayan et al., [CMS Collaboration], J. High Energy Phys. 01079 (2011).

[23] R. Aaji et al., [LHCb Collaboration], Eur.Phys. J. C 74, 2888 (2014).

[24] A. Breakstone et al,[Ames-Bologna-CERN-dortmud-HeidelbergWarsaw Collaboration],Phys.Rev. D 30, 528 (1984).

[25] R. P. Feynman, Phys. Rev. Lett. 23, 1415 (1969). 